\title{
MAMIFEROS DA FAZENDA NHUMIRIM, SUB-REGIÃO DE NHECOLÂNDIA, PANTANAL DO MATO GROSSO DO SUL. I - LEVANTAMENTO PRELIMINAR DE ESPÉCIES *
}

\author{
Cleber J.R. Alho ${ }^{1}$ \\ Thomas E. Lacher, Jr. ${ }^{2}$ \\ Zilca M.S. Campos ${ }^{3}$ \\ Humberto C. Gonçalves ${ }^{3}$
}

\begin{abstract}
The pantanal is one of the world's richest freshwater wetlands. The pantanal is located in the flood plain of the headwaters of the Paraguai river, covering $140,000 \mathrm{~km}^{2}$. The habitats of the Pantanal are present in a complex mosaic. The major habitat types are: pockets of forests, called capao or cordilheira, seasonally flooded grasslands or campos, and permanent or temporary lagoons, called baías. The Pantanal harbors both rich and abundant mammal fauna. A survey was conducted at Fazenda Nhumirim, a research station run by the Centro de Pesquisa Agropecuaria do Pantanal (CPAP) in Corumbá, a research branch of EMBRAPA. The Fazenda covers an area of $4,310 \mathrm{ha}$ in the sub-region of Nhecolândia, appoximately $150 \mathrm{~km}$ east of Corumba, Mato Grosso do Sul. The area receives an average anmual rainfall of $1,022 \mathrm{~mm}$, and has a mean monthly temperature that varies between $29.1^{\circ} \mathrm{C}$ (January) and $22.0^{\circ} \mathrm{C}$ (June). Four routes were followed between two times during ten days of each month. The survey routes were covered on horseback by between two and four observers. Each route was followed and equal number of times in the morning and in the afternoon, to eliminate bias related to the activity patierns of mammals. In order to evaluate relative abundance of nocturnal species, we also conducted a number of nocturnal censuses. These censuses were done by car; a high intensity searchlight was used to spot the animals. We also conducted a trap-mark-recapture survey of the small

1 Centro de Pesquisa Agropecuária do Pantanal e Departamento de Biologia Animal da Universidade de Brasília, 70.910 Brasılia, D.F. Brasil.

2 Huxley College of Environmental Studies, Western Washington University, Bellingham, WA, 98255 E.E.U.U.

3 Instituto de Preservação e Controle Ambiental do Estado do Mato Grosso do Sul, Brasil.

* Trabalho conduzido na Fazenda Nhumirim, Centro de Pesquisa Agropecuária do Pantanal, EMBRAPA, com auxílio da OEA - Organização dos Estados Americanos, através do CNPq, fornecido ao Dr. Cleber J.R. Alho (Conta no 03-85-528-517-BR-1).
\end{abstract}


mammals at Fazenda Nhumirim. After the census period, we continued to collect observations on the occurrence of mammals at the Fazenda, noting wherever possible the kind of habitat in which the animals were observed. The survey identified a diversity of mammals at Fazenda Nhumirim: six orders, 14 families, 19 genera, and 20 species. Nasua nasua was the most frequently observed species during the durnal census, accounting for 61.5 percent of all observations. Dusicyon (formely Cerdocyon) thous was the most frequently observed species on the nocturnal census (39.13\%). The small mammal community of Fazenda Nhumirim is composed of seven species: one marsupial, four cricetine rodents and two echimyid rodents.

\section{INTRODUÇÃO}

O Pantanal é uma das mais ricas terras inundáveis do mundo quanto a abundância e diversidade de fauna. É uma imensa depressão de $140.000 \mathrm{~km}^{2}$ na Bacia do Alto Paraguai. Devido ao relevo da região, os geógrafos dividem a região em três sub-regióes principais: Alto Pantanal, Médio Pantanal e Baixo Pantanal com influência, portanto, no regime de enchente de cada subregião.

Os habitats do Pantanal estão presentes num complexo de recursos distribuídos em mosaico (Lacher \& Alho, no prelo). Há um gradiente norte-sul em grau de inundação com uma mudança correspondente nos tipos de habitats presentes. No Alto Pantanal há muitos rios e corixos que inundam as áreas próximas no período de chuva. Os rios são ladeados por matas ciliares, com densas matas semi-caducifólias em áreas mais elevadas. 0 resto da área é habitat de campo ou campo sazonalmente inundável. Algumas áreas do Pantanal são relacionadas floristicamente ao cerrado do Brasil Central, mas há também influência amazônica. Essa influência se faz mais nítida com a avifauna. O Baixo Pantanal é uma planície sazonalmente inundada com poucos rios ou corixos. Os habitats principais são capơs de matas e "cordilheiras", campos sazonalmente inundáveis, e lagos permanentes ou temporários conhecidos por "baías". O Pantanal do Sul é também relacionado floristicamente com o cerrado, mas não há mais tanta influência de componentes amazônicos. Contudo, há muitas espécies de plantas que são típicas do Chaco.

As oportunidades de observar a rica e abundante mastofauna do Pantanal são propiciadas pela topografia plana e aberta típica da regiáo. Surpreendentemente, pouca pesquisa tem sido feita sobre mamíferos do Pantanal. O trabalho publicado mais ambicioso foi conduzido por Schaller e seus associados no Alto Pantanal. As pesquisas incluíram um levantamento geral e estimativas de densidades de mamiferos grandes (Schaller, 1983), e também estudos mais detalhados sobre a onça (Panthera onca) (Schaller \& Vasconcelos, 1978a ; Schaller \& Crawshaw, 1980, sobre o cervo do Pantanal (Blas- 
tocerus dichotomus) (Schaller \& Vasconcelos, 1978b) e sobre capivara (Hydrochaeris hydrochaeris) (Schaller \& Crashaw, 1981; Alho et al., no prelo).

A ecologia de pequenos mamíferos do Pantanal não foi ainda pesquisada. Schaller (1983) conduziu levantamento (com 1.499 armadilhas-noite em 6 meses) sobre pequenos mamíferos de Acurizal e Alho \& Mares conduziram um levantamento preliminar em Poconé (dados não publicados). Em essência, a fauna de pequenos mamíferos do Pantanal continua desconhecida e sem estudos.

Recentemente um estudo foi iniciado em área florística e faunisticamente diferente: o Pantanal de Nhecolândia. 0 projeto se propõe a conhecer melhor a mastofauna da Fazenda Nhumirim, uma área de 4.310 hectares.

Neste estudo, descreveremos a primeira parte deste trabalho, com um levantamento preliminar dos mamíferos observados. A segunda parte será sobre observaçбes de comportamento, estrutura de grupo, uso de habitat e época de reprodução.

\section{ÁREA DE ESTUDO}

O levantamento foi conduzido na Fazenda Nhumirim, uma estação experimental do Centro de Pesquisa Agropecuária do Pantanal (CPAP) em Corumbá, uma unidade de pesquisa em recursos haturais da EMBRAPA. A Fazenda está localizada a $150 \mathrm{~km}$ este de Corumbá, Mato Grosso do Sul (Lat. $18^{\circ} 59^{\prime} \mathrm{S}$; long. 56 $59^{\circ} \mathrm{O}$ ). Os 4.310 hectares da Fazenda recebem uma precipitação anual média de $1.022,3 \mathrm{~mm}$ e tem uma média de temperatura que varia de $29.1^{\circ} \mathrm{C}$ (janeiro) e $22.0^{\circ} \mathrm{C}$ (junho). $\mathrm{O}$ clima da área foi descrito por Cadavid Garcia, 1984 e EMBRAPA, 1984).

Os habitats da Fazenda Nhumirim foram descritos em detalhe por Alho et al. (no prelo). Resumidamente, a área é formada por campos que são separados por capões de matas semi-caducifólias, cerrado ou cerradão. A área é pontilhada por campo inundável (vazante), "baías" temporárias, e baías permanentes (Fig. 1). A área, como quase todo o Pantanal, é usada para criação extensiva de gado. Os campos são cobertos por espécies forrageiras nativas, mas em algumas áreas há espécies introduzidas.

\section{MÊTODOS}

Uma equipe de pesquisa vem conduzindo pesquisas u campo, com censo demográfico mensal, sobre populaçoes de capivara na Fazenda Nhumirim (Alho et al. no prelo). 0 método usado no censo de outros mamíferos grandes são baseados nessa metodologia de censo de capivara. Quatro roteiros são seguidos duas vezes por dia durante dez dias de cada mês. Cada roteiro cobre 
cerca de 1.070 hectares, representando, portanto, um quarto de toda a área de estudo. Os quatro roteiros cobrem em detalhe toda a área amostrada da Fazenda Nhumirim. Cada roteiro foi seguido igual número de vezes, pela manhã e pela tarde, para eliminar tendenciosidade relacionada ao padrão de atividade dos mamíferos. Como mencionado antes, os roteiros foram estabelecidos por maximizar a exposição de habitats de capivaras, de tal modo que a informação disponível sobre censo de capivara, representa uma estimativa da população total da Fazenda Nhumirim. Como conseqüência, as informaçoes sobre as outras espécies de mamiferos observadas no campo durante os roteiros serão apresentadas num formato de modelo desenvolvido por Eisenberg \& Thorington (1973), onde as informações de censo espécie-específicas sobre números e biomassas são expressas em percentagens dos respectivos totais das espécies observadas. Há técnicas quantitativas disponíveis que poderiam ser também empregadas (veja Glans, 1982) mas ño se mostraram particularmente apropriadas para este censo preliminar executado na Fazenda Nhumirim. Essas técnicas serão discutidas adiante para tentar motivar novos estudos no Pantanal.

Muitas espécies de grandes mamíferos do Pantanal são primariamente noturnas e os procedimentos de censo derivados somente do censo de capivara seriam insuficientes. Para avaliar a abundância relativa de mamíferos noturnos, nós também conduzimos um número de vinte censos noturnos. Esses censos foram feitos a carro, usando um possante refletor manual para localizar os animais ou grupos de animais no campo ou nas periferias dos capóes de mata. As rotas noturnas eram feitas segundo aproximadamente o mesmo roteiro da trilha diurna. A razão disso é que essa trilha podia permitir cobrir uma área de visão grande, contornando as "cordilheiras" de mata, os capбes de cerrado e cerradão e através dos campos entre as "baías". Todos os roteiros cruzavam capōes de cerrado e cerradão e "cordilheiras" de mata semicaducifólia, permitindo observar animais dentro das matas. Os dados sobre o censo noturno estão apresentados no mesmo formato que o censo diurno. As contagens do censo diurno estão disponíveis começando em setembro de 1984 até julho de 1985. Entre setembro e maio, os dados de campo foram coletados principalmente por Campos e Gonçalves, como parte do projeto capivara. As contagens noturnas foram coletadas em junho e julho. Concluído esse período formal de censo, continuamos a coletar dados sonre ocorrência de mamíferos na Fazenda Nhumirim, anotando sempre que possível o tipo

Fig. 1. Vista aérea dos habitats do Pantanal de Nhecolândia, onde os censos de mamíferos foram conduzidos. Esses habitats são formados por capøes de cerrado ou cerradâo, "cordilheiras" de mata semi-caducifólia, campos sazonalmente inundáveis e os lagos localmente chamados de "baías". Os resultados aqui descritos estão baseados em dados produzidos por censos feitos intensivamente em toda a área da Fazenda Nhumirim, com 4.310 hectares. Essa área amostrada por repartida em quatro zonas de censo. Cada rota cobria um quarto da área total e era seguida intensivamente cerca de oito noras por dia durante os 10 primeiros dias de cada mês do ano amostrado. Veja texto para detalhes. 

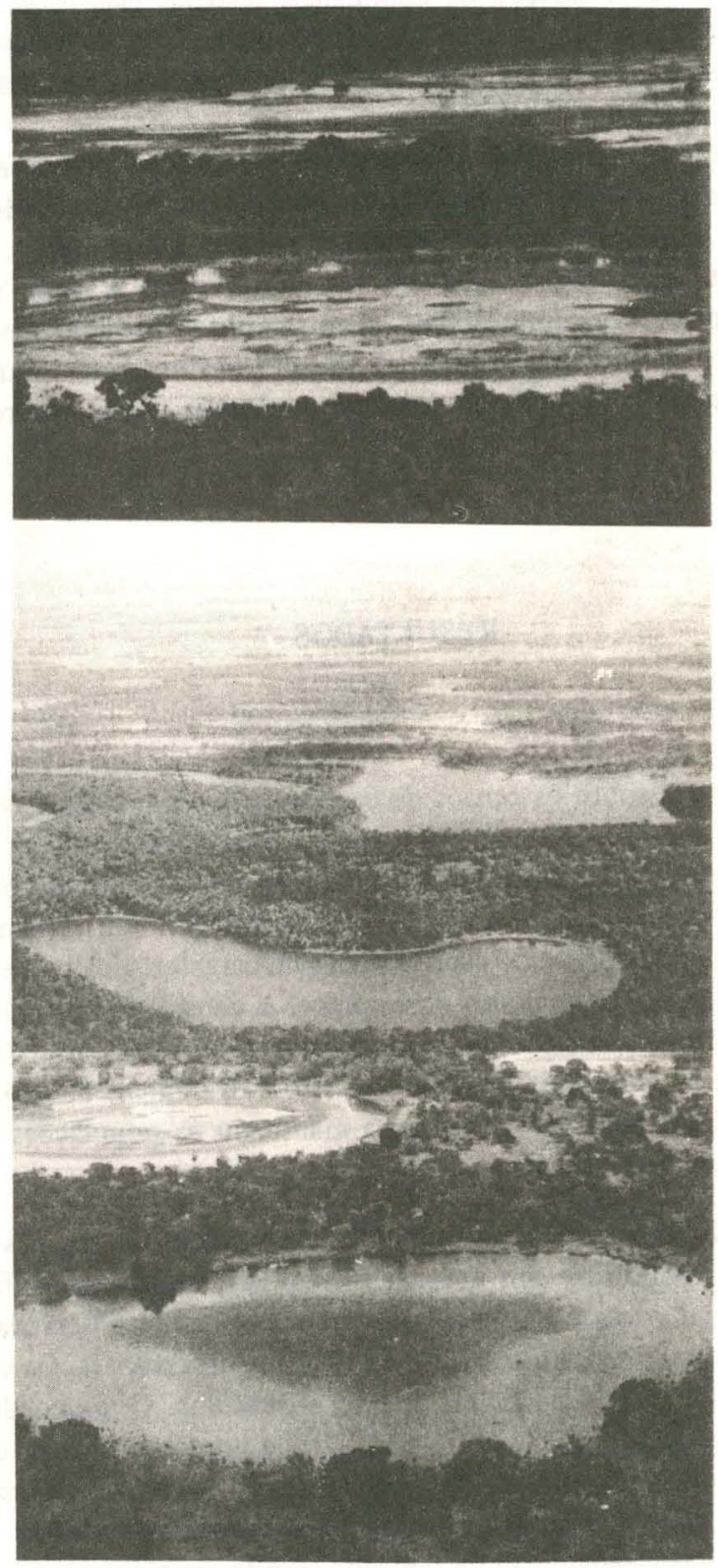
de habitat onde o animal era observado. Foram criados três tipos de formaçð̄es para essas anotaçбes: formação abertas (campo, campo sujo, vazante; capão (cerrado e cerradão) e mate semicaducifólia). Essas informaçðes não foram incluídas na estimação de abundância relativa, mas fornecem dados sobre a área de dispersão nos habitats usados pelas espécies e, também, fornecem informações adicionais sobre algumas espécies mais raras não observadas nos censos formais.

Além disso, conduzimos levantamento especial para pequenos mamiferos. Parte desse levantamento envolve estimativas de denisidade absoluta e avaliação de preferência por microhabitat de espécies residentes. Parte desse levantamento será apresentado em separado (Lacher \& Alho, no prelo). Igualmente armamos algumas linhas adicionais de armadilhas em vários tipos de habitats da Fazenda Nhumirim. Neste trabalho, apresentamos informaçóes sobre sucesso de captura, densidades relativas e biomassa relativa.

\section{RESULTADOS}

Os resultados sobre os censos de capivara não estao incluídos no levantamento geral, mas apresentados separadamente na Tabela 1. Uma média de 215,8 observaçбes sobre capivaras foi feita por mês na Fazenda Nhumirim. Esse número atingiu um máximo de 255 em setembro e um mínimo de 154 em fevereiro. As populaçбos de capivara atingem caracteristicamente uma baixa durante a estação cheia, como observamos. A densidade bruta para a área total da Fazenda não considerando preferência de habitat foi de 0,07 capivaras por hectare. A densidade ecológica, isto é, o número de capivaras encontradas em áreas mais vantajosas para manterem as populaçбes tem uma média de 0,14 capivaras por hectare.

Através do censo identificamos uma diversidade de grandes mamíferos na Fazenda Nhumirim: seis ordens, 14 famılias, 19 gêneros e 20 espécies (Tabela 2). Nasua nasua é a espécie mais freqüentemente observada durante o censo diurno, representando $61,5 \%$ de todas as observaçóes. Os coatis são gregários e barulhentos, sendo facilmente observados. 0 porco doméstico que se torna selvagem (chamado no Pantanal de porco-monteiro), é a próxima espécie mais freqüentemente observada (9,39\%), seguida de Mazama americana $(8,45 \%)$, Alouatta caraya $(4,46 \%)$ e Ozotocerus bezoarticus $(4,46 \%)$. Sem surpresa, essas espécies são de tamanho grande, gregárias e vocais, permitindo a observação. Espécies como Euphractus sexcinctus e Dasypus novemcinctus foram sempre subestimados, por causa do tamanho pequeno e seu hábito silencioso. 
Tabela 1 - Número de capivaras observadas e tamanho médio de grupo ( \pm 1 desvio padrão) para 12 meses de levantamento. Trinta e oito grupos estavam presentes no levantamento. A área da Fazenda é de 4.310 ha.

\begin{tabular}{lcc}
\hline \multicolumn{1}{c}{ Mês } & Número de Observação & Tamanho Médio do Grupo \\
\hline outubro & 252 & $6.6 \pm 4.9$ \\
novembro & 239 & $6.3 \pm 4.3$ \\
dezembro & 226 & $5.9 \pm 4.2$ \\
janeiro & 193 & $5.1 \pm 3.4$ \\
fevereiro & 154 & $4.0 \pm 2.5$ \\
março & 162 & $4.3 \pm 2.6$ \\
abril & 193 & $5.1 \pm 3.9$ \\
maio & 192 & $5.0 \pm 3.7$ \\
junho & 250 & $6.6 \pm 4.7$ \\
julho & 211 & $5.6 \pm 4.8$ \\
agosto & 251 & $6.6 \pm 5.2$ \\
setembro & 266 & $7.0 \pm 5.3$ \\
\hline
\end{tabular}

Dusicyon (formalmente Cerdocyon) thous foi a espécie mais freqüentemente observada no censo noturno $(39,13 \%)$ seguido de Mazama america$n a(36,96 \%)$, veja Tabela 2 . As outras espécies eram observadas com pouca freqüência. Os resultados numéricos dos censos diurnos e noturnos refletem duas coisas: abundância relativa das espécies e espécies mais facilmente observadas. Nem todas as espécies são igualmente suscetiveis de observação através de censos visuais (Cant, 1977) e esses censos devem ser analisados dentro dos limites de suas contribuiçбes.

Considerando que as espécies variam em peso, o número de indivíduos observados não fornece uma boa estimativa da importância relativa das espécies no ecossistema. É comum fornecer os resultados de censos em termos de biomassa (Eisenberg \& Thorington 1973; Eisenberg et al., 1979; Schaller, 1983). Para cumprir esse procedimento é necessário estimar a massa média de cada espécie. Essa massa média poderia refletir não somente o peso dos animais adultos, mas também jovens. A média representa o peso aproximado do "indivíduo médio" na população de classes etárias mistas. Essas estimativas já estão disponíveis para todas as espécies que observamos no censo, exceto para Sus scrofa; veja Schaller (1983). O peso médio do porco varia muito na literatura. Baseados em nossas observaçðes sobre o tamanho e classe de idade de Sus na Fazenda Nhumirim, estabelecemos um peso médio de $30 \mathrm{~kg}$.

Há espécies dominantes nas biomassas dos censos diurnos e noturnos (Tabela 3). O porco monteiro é a espécie mais importante no censo diurno $(28,97 \%)$ seguida do coati Nasua $(18,97 \%)$, do veado campeiro $(18,35 \%)$ e veado mateiro Mazama $(13,04 \%)$. Mazama americana é a espécie mais dominante no censo noturno (48,5\%), seguida de Sus $(33,82 \%)$ e Dusicyon $(17,8 \%)$. Os dados de censo, avaliados para biomassa, têm as mesmas limita- 
ções que os dados numéricos. Contudo, esses dados revelam situaçōes impos tantes. A mais importante talvez seja o presente papel desempenhado po Sus scrofa no ecossistema, principalmente lembrando que é uma espécie in troduzida e que ocupa em abundância o nicho de espécies de hábitos e habitats semelhantes. Contribui, no momento, com uma importante percentagem de biomassa, menor somente que a de capivara. Em contraste, os porcos endêmicos Tayassu tajacu e T. Pecari são mais raros. Aparentemente há competição entre as três espécies, com a invasão de nichos originais das espécies da fauna local pela espécie introduzida. Contudo, precisa-se de pesquisa especialmente planejada para investigar esses aspectos de ecologia. Temos observado predação esporádica de filhotes e ovos de animais silvestres por porco monteiro. Por exemplo, alguns porcos são capazes de localizar e predar ovos de jacarés encontrados em ninhos localizados nos capões de mata. Um de nós (Z. Campos) observou predação de capivara muito jovem por porco monteiro.

Tabela 2 - Resultados preliminares de censo de mamíferos (exceto pequenos) conduzidos na Fazenda Nhumirim. As observações de censo diurno cobrem o período de setembro de 1984 a julho de 1985 . O censo noturno é de junho e julho de 1985 . Os dad apresentam número total de observações e percentagem total de observação.

\begin{tabular}{|c|c|c|c|c|}
\hline Espécies & $\begin{array}{c}\text { Censo Diurno } \\
\text { Número de } \\
\text { Observação }\end{array}$ & $\%$ & $\begin{array}{c}\text { Censo Noturno } \\
\text { Número de } \\
\text { Observação }\end{array}$ & $\%$ \\
\hline Myrmecophaga tridactyla & 3 & 0.70 & 0 & 0.00 \\
\hline Tamandua tetradactyla & 1 & 0.23 & 1 & 2.17 \\
\hline Euphractus sexcinctus & 11 & 2.58 & 0 & 0.00 \\
\hline Dasypus novemcinctus & 1 & 0.23 & 1 & 2.17 \\
\hline Alouatta caraya & 19 & 4.46 & 0 & 0.00 \\
\hline Dasyprocta punctata & 5 & 1.17 & 0 & 0.00 \\
\hline Hydrochaeris hydrochaeris & - & 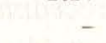 & - & - \\
\hline Thrichomys apereoides & 0 & 0.00 & 1 & 2.17 \\
\hline Nasua nasua & 262 & 61.50 & 0 & 0.00 \\
\hline Procyon cancrivorous & 1 & 0.23 & 3 & 6.52 \\
\hline Eira barbara & 3 & 0.70 & 0 & 0.00 \\
\hline Dusicyon thou & 13 & 3.05 & 8 & 39.13 \\
\hline Felis concolor & 1 & 0.23 & 0 & 0.00 \\
\hline Felis pardalis & 1 & 0.23 & 0 & 0.00 \\
\hline Tapirus terrestri & 2 & 0.47 & 0 & 0.00 \\
\hline Sus scrofa & 40 & 9.39 & 4 & 8.70 \\
\hline Tayassu tajac. & 7 & 1.64 & 0 & 0.00 \\
\hline Blastocerus dichotom. & 1 & 0.23 & 0 & 0.00 \\
\hline Ozotoceros bezoartic $u$ & 19 & 4.46 & 1 & 2.1 \\
\hline Mazama americān & 36 & 8.45 & 17 & 36.96 \\
\hline Total & 426 & 100 & 46 & 100 \\
\hline
\end{tabular}

* Nados sobre capivara disponíveis em Alho, et al. (no prelo) 
Tabela 3 - Resultados preliminares de censo de mamíferos conduzidos sa Fazenda Nhumirim. Os dados de biomassa foram feitos segundo Schaller (1983). O período de censo é o mesmo da Tabela 4.

\begin{tabular}{|c|c|c|c|c|c|}
\hline Espécies & $\begin{array}{l}\text { Biomas- } \\
\text { sa média } \\
\text { (kg) }\end{array}$ & $\begin{array}{l}\text { Censo } \\
\text { Diurno } \\
\text { Biomassa } \\
\text { (kg) }\end{array}$ & $\begin{array}{c}\% \text { de } \\
\text { Biomas- } \\
\text { sa }\end{array}$ & $\begin{array}{c}\text { Censo } \\
\text { Noturno } \\
\text { Biomassa } \\
(\mathbf{k g})\end{array}$ & $\%$ \\
\hline Myrmecophaga tridactyla & 20.00 & 60.00 & 1.45 & 0.00 & 0.00 \\
\hline Tamandua tetredactyla & 5.00 & 5.00 & 0.12 & 5.00 & 0.95 \\
\hline Euphractus sexcinctus & 3.30 & 36.30 & 0.88 & 0.00 & 0.00 \\
\hline Dasypus novemcinctus & 3.00 & 3.00 & 0.07 & 3.00 & 0.57 \\
\hline Alouatta caraya & 4.50 & 85.50 & 2.06 & 0.00 & 0.00 \\
\hline Dasyprocta punctata & 3.00 & 15.00 & 0.36 & 0.00 & 0.00 \\
\hline Hydrochaeris hydrochaeris * & 30.00 & - & - & - & - \\
\hline Thrichomys apereoides & 0.20 & 0.00 & 0.00 & 0.20 & $0.00 *$ \\
\hline Nasua nasua & 3.00 & 786.00 & 18.97 & 0.00 & 0.00 \\
\hline Procyon cancrivorous & 10.00 & 10.00 & 0.24 & 9.00 & 1.71 \\
\hline Eira barbara & 4.00 & 13.00 & 0.29 & 0.00 & 0.00 \\
\hline Dusicyon thous & 5.20 & 67.60 & 1.63 & 93.60 & 17.80 \\
\hline Felis concolor & 48.00 & 48.00 & 1.16 & 0.00 & 0.00 \\
\hline Felis pardalis & 8.00 & 8.00 & 0.19 & 0.00 & 0.00 \\
\hline Tapirus terrestris & 150.00 & 300.00 & 7.24 & 0.00 & 0.00 \\
\hline Sus scrofa & 30.00 & 1200.00 & 28.97 & 120.00 & 22.82 \\
\hline Tayassu tajacu & 18.00 & 126.00 & 3.04 & 0.00 & 0.00 \\
\hline Blastocerus dichotomus & 80.00 & 80.00 & 1.93 & 0.00 & 0.00 \\
\hline Ozotoceros bezoarticus & 40.00 & 760.00 & 18.35 & 40.00 & 7.61 \\
\hline Mazama americana & 15.00 & 540.00 & 13.04 & 255.00 & 48.50 \\
\hline Total & & 4142.00 & $100 \%$ & & $100 \%$ \\
\hline
\end{tabular}

* As percentagens são muito baixas para serem indicadas.

Tabela 4 - Número relativo e biomassa de pequenos mamíferos capturados na Fazenda Nhumirim. Os pesos das espécies são estimados da literatura e deste estudo. A biomassa é expressa em gramas.

\begin{tabular}{lcrrrr}
\hline \multicolumn{1}{c}{ Espécie } & $\begin{array}{c}\text { Número de } \\
\text { Captura }\end{array}$ & $\%$ & $\begin{array}{c}\text { Biomassa } \\
\text { Média }\end{array}$ & $\begin{array}{c}\text { Biomassa } \\
\text { Total }\end{array}$ & $\%$ \\
\hline Monodelphis domestica & 04 & 2.41 & $75^{1}$ & 300 & 2.21 \\
Calomys callosus & 03 & 1.81 & $30^{2}$ & 90 & 0.66 \\
Oryzomys fornesi & 78 & 46.99 & $12^{3}$ & 936 & 6.89 \\
O. concolor & 05 & 3.01 & $50^{2}$ & 250 & 1.84 \\
O. subflavus & 37 & 22.29 & $75^{3}$ & 2775 & 20.44 \\
Clyomys laticeps & 20 & 12.05 & $200^{3}$ & 4000 & 29.46 \\
Thrichomys apereoides & 19 & 11.45 & $275^{2}$ & 5225 & 38.49 \\
\hline Total & 166 & 100 & - & 13576 & 100 \\
\hline
\end{tabular}

Citações: 1) Fadem et al. (1982); 2) este estudo; 3) Mares et al. 
Nosso rápido censo de pequenos mamíferos revelou uma comunidade ecológica composta por sete espécies: um marsupial, quatro roedores cricetideos e dois roedores equimiídeos (Tabela 4). Os resultados da Tabela 4 são baseados em 3.582 armadilhas/noite perfazendo 166 capturas com um sucesso de captura de $4,63 \%$. A espécie de roedor mais importante capturada foi Oryzomys fornesi, um cricetíneo pequeno de rabo longo. Oryzomys fornesi, em todos os habitats exceto em mata semi-caducifólia, constituindo-se na espécie mais generalista capturada. Outra espécie de cricetineo de tamanho maior, Oryzomys subflavus, foi a outra espécie mais comum, vivendo em capão de cerrado. As duas espécies de roedores equimiídeos abundantes foram Thrichomys apereoides e Clyomys laticeps. Os trabalhos anteriores se referem a $T$. apereoides como vivendo em habitats rupestres do cerrado. No Pantanal são localmente abundantes em capões de cerrado de solo arenoso sem qualquer pedra no habitat. Clyomys laticeps é também abundante em bordas de capóes e em campo. Esta espécie é muito pouco estudada. No Pantanal vivem em colônias, em sistemas de tocas em capóes de cerrado ralo ou em campo nas proximidades de capðes. Capturamos essa espécie com bastante freqüência em campos de roça de cultivo de mandioca e milho. Nesses loçais extendem o sistema de tocas e galerias até as raízes da mandioca para consumi-las. As outras três espécies de roedores coletados eram raros. As comparações de biomassa mostram um padrão diferente de abundância relativa (Tabela 4). Claramente, quanto maior a espécie é, mais importante aparece, embora ñ̃o seja a mais comum. Os dois equimiídeos assumem a liderança em primeiro e segundo lugares. Oryzomys fornesi é considerada a espécie mais importante quando considerada em abundância numérica. Cai, no entanto, de importância, quando a comparação é baseada em biomassa, devido a seu tamanho pequeno, atingindo $40,1 \%$.

$\mathrm{O}$ número total de mamíferos médios e grandes observados durante um período de 22 meses está representado na Tabela 5. Mamíferos pequenos e capivaras não estão incluídos nessa lista. Para informação sobre capivaras veja Alho et al., no prelo. Em 22 meses, observamos um total de 1.243 mamiferos. Certamente, muitos indivíduos foram observados mais de uma vez. A Tabela 5 dá uma idéia da facilidade de vezes com que algumas espécies são observadas, enquanto outras são mais difíceis. A maioria das espécies foi observada em formações abertas, como os campos, principalmente porque nessas formaçбes os animais são mais facilmente observados quando saem para forragear ou beber. Além de os campos em volta das baías fornecerem forragem para os herbívoros, fornecem também invertebrados para mamíferos de diferentes dietas, como caranguejos, caramujos, formigas e cupins para Dusicyon, Procyon, Nasua, Sus e as duas espécies de Tayassu.

Preparamos uma lista provisória de mamíferos da Fazenda Nhumirim, baseada nos levantamentos das capturas adicionais por armadilhas e em outras observaçōes fora dos roteiros (Tabela 6). Há 34 espécies presentes nessa lista. Algumas dessas espécies precisam de verificação taxonômica e a lista não pre- 
tende ser completa. Os gêneros de roedores Akodon, Bolomys, Oxymycterus e Scapteromys estđo ausentes nessas áreas amostradas. Algumas espécies listadas precisam de mais estudo intensivo quanto, inclusive, a identificação, como Mazama rufa e Mazama gouazoubira.

Tabela 5 - Número de observações de espécies de mamíferos dentro e fora das linhas de censo.

Tipo de habitat

Espécie

$\begin{array}{lll}\text { Nome comum } & \begin{array}{l}\text { Campos Capão } \\ \text { vazante }\end{array} \text { lheira }\end{array}$

\begin{tabular}{|c|c|c|c|c|}
\hline Myrmecophaga tridactyla & Tamanduá bandeira & 10 & 2 & 2 \\
\hline Tamandua tetradactyla & Tamanduá mirim & 6 & 2 & 1 \\
\hline Euphractus sexcinctus & Tatu peba & 19 & 6 & - \\
\hline Dasypus novemcinctus & Tatu galinha & 5 & - & - \\
\hline Tolypeutes matacus & Tatu bola & 1 & - & - \\
\hline Priodontes maximus & Tatu canastra & - & 1 & - \\
\hline Alouatta caraya & Bugio & - & 5 & 44 \\
\hline Dasyprocta punctata & Cutia & 3 & 12 & 6 \\
\hline Nasua nasua & Coati & 561 & 93 & 26 \\
\hline Procyon cancrivorous & Mão-pelada & 22 & 2 & - \\
\hline Eira barbara & Irara & 7 & - & - \\
\hline Dusicyon thous & Lobinho & 85 & 9 & 4 \\
\hline Speothos venaticus & Cachorro vinagre & - & 2 & 2 \\
\hline Felis concolor & Onça parda & 7 & 4 & - \\
\hline Felis yagouarond $i^{*}$ & Gato mourisco & 1 & - & - \\
\hline Felis pardalis & Jaguatirica & 1 & 1 & - \\
\hline Tapirus terrestris & Anta & 7 & 1 & 7 \\
\hline Sus scrofa & Porco-monteiro & 70 & - & 1 \\
\hline Tayassu tajacu & Cateto & 12 & 1 & 3 \\
\hline Tayassu pecari & Queixada & 33 & - & - \\
\hline Blastocerus dichotomus & Corvo-do-Pantanal & 4 & - & - \\
\hline Ozotoceros bezoarticus** & Veado campeiro & 45 & 2 & - \\
\hline Mazama americana & Veado mateiro & 69 & 41 & 1 \\
\hline Mazama rufa & Veado bororó & - & 1 & - \\
\hline Mazama gouazoubira & Veado virá & 2 & - & - \\
\hline
\end{tabular}

* Visto uma vez fora da Fazenda, na rota para Corumbá.

**A maioria das observações aqui foi em campos entre Nhumirim e Campo Dora.

\section{DISCUSSÃO}

Este censo preliminar revelou que o Pantanal de Nhecolândia, amostrado na Fazenda Nhumirim, alberga uma fauna diversa de mamíferos. Há, como apontado, limitaçбes de informações oriundas do método empregado. Nesta e em outras áreas, um levantamento mais intensivo e cobrindo maior espaço de tempo deverá ser empregado. Há certas metodologias já consagra- 
Tabela 6 - Mamíferos da Fazenda Nhumirim. Esta lista combina dados do censo geral, censo de pequenos mamíferos e dados adicionais fora das linhas de censo. A lista está em ordem, família, gênero e espécie. A classificação é feita segundo Honaki et al. (1982)

Marsupialia

Didelphidae Monodelphis domestica Didelphis albiventris Marmosa sp. *

Primates

Cebidae Alouatta caraya

Edentata

Myrmecophagidae

Myrmecophaga tridactyla

Tamandua tetradactyla

Dasypodidae

Euphractus sexcinctus

Dasypus novemcinctus

Tolypeutes matacus

Priodontes maximus
Hydrochaeridae

Hydrochaeris hydrochaeris

Carnivora

Procyonidae

Nasua nasua

Procyon cancrivorus

Mustelidae

Eira barbara

Canidae

Dusicyon thous

Speothos venaticus

Felidae

Felis pardalis

Felis concolor
Perissodactyla

Tapiridae

Tapirus terrestris

Artiodactyla

Suidae

Sus scrofa

Tayassuidae

Tayassu pecari

Tayassu tajacu

Cervidae

Mazama rufa *

Mazama gouazoubira*

Mazama americana

Ozotoceros bezoarticus

Blastocerus dichotomus

Espécie que precisa de maior atenção taxonômica. 
das que podem ser sugeridas como aquelas encontradas em Kelker (1945) e Anderson \& Pospahala (1970).

Outro projeto que precisa investigação mais profunda é sobre ecologia de população de Sus scrofa e o impacto desses porcos-monteiros sobre espécie nativas como Tayassu tajacu e $T$. pecari. Enfase especial sobre competição por recursos (espaço, alimentos, abrigo, área de reprodução) entre essas três espécies simpátricas deve ser considerada. Aparentemente o crescimento de populaçбes de porco monteiro vem inibindo e deslocando o tamanho de população de queixada e cateto. $O$ fato de uma espécie introduzida constituir-se com componente importante da comunidade ecológica (Tabela 3) é causa de preocupação. Os habitats do Pantanal têm sofrido um processo de fragmentação devido à atividade humana o que torna os estudos de ecologia mais urgentes. Essa prioridade de pesquisa há de ser transformada em ação com estudos em preferência de habitats, sistemática, uso de espaço, radiação de espécie e história natural para melhor entendimento desse bioma tão importante.

\section{AGRADECIMENTOS}

Os autores agradecem a Araê Book e a Kazuyoshi Ofugi as facilidades criadas para tornar este trabalho possível no CPAP. Muitas pessoas na Fazenda Nhumirim participaram entusiasticamente dos trabalhos de campo, quer seguindo as rotas de censo, quer ajudando a armar as armadilhas, entre estes Eleutério P. Santos, Nauílio A. Costa e Ernande Ravaglia. A OEA concedeu ajuda em nome de um dos autores (Dr. Alho) que tornou possível a participação de $\mathrm{T}$. Lacher Jr. $\mathrm{O}$ CNPq também concedeu ajuda à pesquisa. Lacher Jr. agradece o apoio logístico do Bureau for Faculty Research da Western Washington University. Gonçalves e Campos tiveram apoio do Instituto de Preservação Ambiental - INAM-SEMA, do Mato Grosso do Sul.

\section{REFERÊNCIAS}

Alho, C.J.R.; Campos, Z.M.S. e Gonçalvez, H.C. Ecologia de capivara (Hydrochaeris hydrochaeris, Rodentia) do Pantanal: I - habitat, densidades e tamanho do grupo. Revista Brasileira de Biologia. No prelo.

Alho, C.J.R.; Campos, Z.M.S. e Gonçalves, H.C. Ecologia de capivara (Hydrochaeris hydrochaeris, Rodentia) do Pantanal: II - atividade sasonalidade, uso do espaço e manejo. Revista Brasileira de Biologia. No prelo.

Alho, C.J.R. 1986. Capivaras: uma vida em familia. Ciência Hoje. Sociedade Brasileira para o Progresso da Ciência. Vol. 4. no 23:64-68. 
Anderson, D.R. e R.S. Pospahala. (1970). Correction of bias in belt transect studies of immobile objects. J. Wildl. Manag. 34: 141-146.

Cadavid Garcia, E.A. 1984. O clima do Pantanal. Corumbá, EMBRAPA, UEPAE de Corumbá, Circular Técnica 14, 39 pp.

Cant. J.G.H. (1977). A census of the agouti (Dasyprocta punctata) in seasonally dry forest at Tikal, Guatemala, with some comments on strip censusing. J. Mamm. 58: 688-690.

Eisenberg, J.F., M.A. O'Conell, e P.V. August. (1979). Density, productivity, and distribution of mammals in two Venezuela habitats. In. J.F. Eisenberg (ed.). Vertebrate Ecology in the Northern Neotropics. p.p. 187-207. Smithsonian Institution Press, Washington, D.C.

Eisenberg, J.F. e T.W. Thorington, J.R. (1973). A preliminary analysis of a neotropical mammal fauna. Biotropica 5: 150-161.

EMBRAPA. 1984. Boletim Agrometeorológico: cinco anos de observações meteorológicas, Corumbá, MS, 1977-1981. Corumbá, EMBRAPA, UEPAE de Corumbá, 52 pp.

Fadem, B.H., G.L. Trupin, E. Malianiak, J.L. Vandeberg, e V. Hayssen. 1982. Care and breding of the gray, short-tailed opossum (Monodelphis domestica), Lab. Anim. Sci.32: 405-409.

Glans, W.E. (1982). The terrestrial mammal fauna of Barro Colorado Island: censures and long-term changes. In E.G. Laigh, Jr., A.S. Rand. \& D.M. Windsor (eds.). The Ecology of a Tropical Forest. pp. 455-468. Smithsonian Institution Press, Washington, D.C.

Honacki, J.H., K.E. Kinman, \& J.W. Koeppl. (1982). Mammal Species of the World. Allen Press, Lawrence.

Kelker, G.H. (1945). Measurement and interpretation of forces that determine population of managed deer. Ph. D. Thesis. Univ. of Michigan, ann Arbor.

Lacher, T.E. Jr., \& C.J.R. Alho. (no prelo). Densities and microhabitat preferences of the mammals of Fazenda Nhumirimm, Sub-region Nhecolândia, Pantanal of Mato Grosso do Sul. Ciência Interamericana.

Schaller, G.B. (1983). Mammals and their biomass on a Brazilian ranch. Arqos. Zool., S. Paulo 31 (1): 1-36.

Schaller, G.B. \& P.G. Crzwshaw Jr. (1980). Movement patterns of jaguar. Biotropica 12 (3): 161-168.

Schaller, G.B. \& J.M.C. Vasconcelos. (1978a). Jaguar predation on capybara. Z. Säugertierk. 43 (5): 296-301.

Schaller, G.B. \& Y. M.C. Vasconcelos. (1978b). A marsh deer census in Brazil. Oryx 14 (4): $345-351$. 